\title{
OPTICAL AMPLIFICATION IN AVIONIC ANALOG PHOTONIC LINKS
}

\author{
Vincent J. Urick and Frank Bucholtz \\ U.S. Naval Research Laboratory, Optical Sciences Division, Washington, DC 20375
}

\section{Introduction}

Analog photonic links are a promising technology for the avionic platform. Fiber-optic cabling is invulnerable to electromagnetic interference and is capable of carrying essentially any range of radio, microwave or millimeter-wave frequencies. In addition, fiber-optic cables offer reduced size and increased flexibility as compared to typical RF cabling. While the fiber-optic cabling itself has low loss, typically $0.0005 \mathrm{~dB} / \mathrm{m}$ for the signal, the electrical-optical-electrical conversion can be quite lossy. However, high-performance analog links have been demonstrated with net electronic gain and acceptable electronic noise figure [1],[2]. Such links require a combination of high-power low-noise optical sources, low- $\mathrm{V}_{\pi}$ modulators and high-current photodetectors. These high-optical-power links are particularly attractive for avionic platforms that consist of short transmission spans on the order of 10's or 100's of meters where fiber nonlinearities are negligible. High-current photo-detectors [3] and low- $\mathrm{V}_{\pi}$ modulators [4] have been demonstrated and are available in relatively small packages. However, high-power lasers have yet to be demonstrated in a size acceptable for avionics applications. Here we report on the use of highly saturated erbium-doped fiber amplifiers (EDFAs) and moderate-power lownoise lasers in short analog photonic links to replace high-power low-noise lasers. Using the concepts of generalized relative intensity noise and noise penalty [5], we calculate system performance capabilities and demonstrate them experimentally. It is shown that an amplified link can achieve nearshot-noise-limited performance.

\section{Noise Penalty in IMDD Systems}

For an analog photonic link, we cite the four most important performance metrics as electronic gain $\left(G_{\mathrm{rf}}\right)$, electronic noise figure $\left(N F_{\mathrm{rf}}\right)$, spuriousfree dynamic range $(S F D R)$ and 1-dB compression dynamic range $(C D R)$. For an intensity-modulation direct-detection (IMDD) link these metrics are

$$
G_{\text {rf }}=\left(I_{\mathrm{dc}} \pi / V_{\pi}\right)^{2} Z_{\text {in }} Z_{\text {out }}
$$

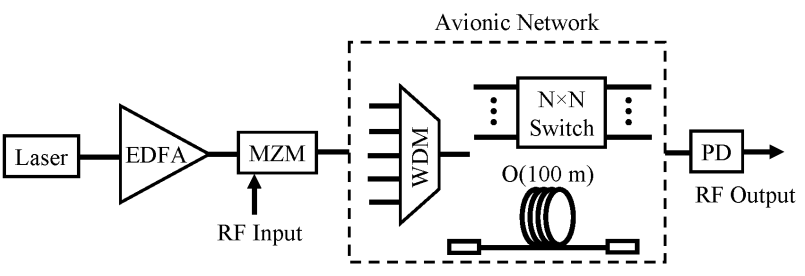

Figure 1. Optically-Amplified Avionic Photonic Link

$$
\begin{gathered}
N F_{\mathrm{rf}}=R I N_{\text {total }} V_{\pi}^{2} /\left(\pi^{2} k_{\mathrm{B}} T Z_{\text {in }}\right) \\
S F D R=\left(4 / R I N_{\text {total }}\right)^{2 / 3} \\
C D R=\phi_{\text {1dB }}^{2} /\left(2 R I N_{\text {total }}\right)
\end{gathered}
$$

where $I_{\mathrm{dc}}$ is the DC photocurrent, $Z_{\text {in }}$ and $Z_{\text {out }}$ the input and output impedances, $k_{\mathrm{B}}$ Boltzmann's constant, $T$ the temperature, $\phi_{1 \mathrm{~dB}}$ the phase-shift amplitude for $1-\mathrm{dB}$ of compression and $R I N_{\text {total }}$ the total output-referenced generalized relative intensity noise. Here $R I N_{\text {total }} \equiv N_{\text {out }} /\left(I_{\mathrm{dc}}^{2} Z_{\text {out }}\right)$ where $N_{\text {out }}$ is the output-reference noise power spectral density. In an optically-amplified short-length analog photonic link, such as shown in Fig. 1, the noise penalty incurred $(N P)$ by employing an EDFA as compared to an unamplified system can be written as

$$
N P \equiv 1+\frac{R I N_{\text {sig-sp }}+R I N_{\text {sp-sp }}}{R I N_{\text {shot }}+R I N_{\text {ith }}+R I N_{\text {oth }}+R I N_{\text {laser }}}
$$

where RIN associated with signal-spontaneous beating $\left(R I N_{\text {sig-sp }}\right)$, spontaneous-spontaneous beating $\left(R I N_{\text {sp-sp }}\right)$, shot noise $\left(R I N_{\text {shot }}\right)$, input $\left(R I N_{\text {ith }}\right)$ and output $\left(R I N_{\text {oth }}\right)$ thermal noise, and laser noise $\left(R I N_{\text {laser }}\right)$ are considered. It can be seen that the penalties in $N F_{\mathrm{rf}}, S F D R$ and $C D R$ are equal to $N P$, $(2 / 3) N P[\mathrm{~dB}]$ and $N P$, respectively.

Except for $R I N_{\text {laser, which we treat as a }}$ measurable quantity, we have for the terms in (5),

$$
R I N_{\text {sig-sp }}=2 h v N F_{\text {opt }} / P_{\text {in }}
$$




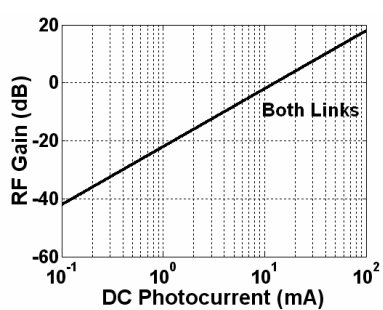

(a)

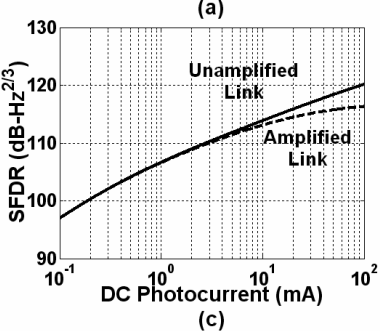

(c)

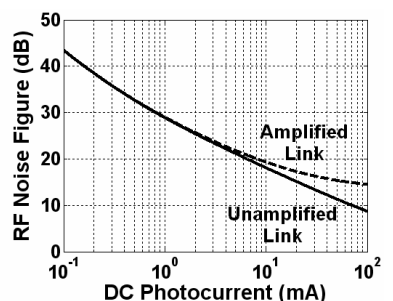

(b)

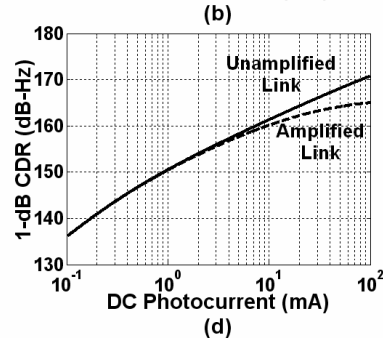

(d)

Figure 2. Calculated IMDD Link Performance

$$
\begin{gathered}
R I N_{\mathrm{sp}-\mathrm{sp}}=\left(h v N F_{\mathrm{opt}} / P_{\mathrm{in}}\right)^{2} B_{\mathrm{opt}} \\
R I N_{\mathrm{shot}}=2 h v /\left(\eta G_{\mathrm{opt}} l_{\mathrm{opt}} P_{\mathrm{in}}\right) \\
R I N_{\text {ith }}=\pi^{2} k_{\mathrm{B}} T Z_{\mathrm{in}} / V_{\pi}^{2} \\
R I N_{\mathrm{oth}}=k_{\mathrm{B}} T /\left(I_{\mathrm{dc}}^{2} Z_{\mathrm{out}}\right)
\end{gathered}
$$

where $h$ is Planck's constant, $v$ is the optical frequency, $N F_{\text {opt }}$ is the EDFA noise figure, $P_{\text {in }}$ is the input power to the EDFA, $B_{\mathrm{opt}}$ is the optical bandwidth, $\eta$ is the detector quantum efficiency, $G_{\text {opt }}$ is the EDFA optical gain (assumed to be much larger than unity), and $l_{\text {opt }}$ is the post-EDFA optical loss. In deriving (7), it was assumed that $B_{\mathrm{rf}}, B_{\mathrm{opt}}$ $>>f_{\mathrm{rf}}$ where $B_{\mathrm{rf}}$ is the RF bandwidth and $f_{\mathrm{rf}}$ is the RF frequency of interest.

The calculated performance of an amplified (dashed) and unamplified (solid) IMDD analog link is shown in Fig. 2. Equations (1)-(4) and (6)-(10) were used with $v=193 \mathrm{THz}, N F_{\text {opt }}=3 \mathrm{~dB}, P_{\text {in }}=50$ $\mathrm{mW}, B_{\text {opt }}=3 \mathrm{THz}$ (25 nm in wavelength), $T=300$ $\mathrm{K}, V_{\pi}=2 \mathrm{~V}, Z_{\text {in }}=Z_{\text {out }}=50 \Omega$ and $R I N_{\text {laser }}=-190$ $\mathrm{dBc} / \mathrm{Hz}$. At high photocurrents the unamplified link performance is limited by shot noise whereas the amplified link is limited by signal-spontaneous beating. In these limits $\mathrm{NP} \rightarrow 0 \mathrm{~dB}$ when $l_{\text {opt }}<<$ $\left(1 / \eta G_{\text {opt }} N F_{\text {opt }}\right)$, which describes cases where EDFAs can be used without any performance penalties.

In practice $N F_{\text {opt }}$ is a function of $P_{\text {in }}$ and we show in Fig. 3 the measured performance of a particular EDFA (Avanex Model 03-0905-00-01) in

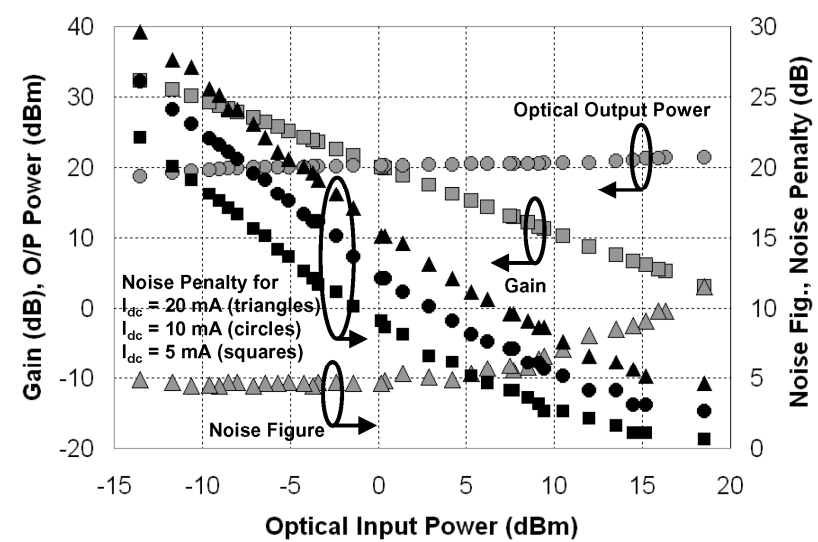

Figure 3. Measured IMDD Link Performance

a link similar to that in Fig. 1. For these data an optical attenuator was employed to simulate avionic network loss. A low-noise laser (CLR Photonics METEOR $^{\circledR}$ ) was used as the seed, which has a measured $R I N_{\text {laser }}=-190 \mathrm{dBc} / \mathrm{Hz}$ at $f_{\mathrm{rf}}=1 \mathrm{GHz}$ where the measurements were made. Note that $N P$ can be quite low even at high $I_{\mathrm{dc}}$ as long as the EDFA is adequately saturated.

\section{Conclusions}

We have described cases where the penalties incurred in IMDD links by utilizing EDFAs can be quite small or negligible. These conclusions are particularly important for short-length high-opticalpower links, such as can be used in an avionic platform. These results relax the output-power requirements on low-noise lasers. In addition, this work demonstrates the significance of a new class of EDFAs, namely, EDFAs in compact flightqualified packages having low noise figure in deep saturation.

\section{References}

[1] C. H. Cox III, et al., "Limits on the performance of RFover-fiber links and their impact on device design," IEEE Trans. Microwave Theory Tech., vol. 54, no. 2, pp. 906-920, Feb. 2006.

[2] V. J. Urick, et al., "Wideband (0.045-6.25 GHz) 40-km analog fiber-optic link with ultra-high $(>40 \mathrm{~dB})$ all-photonic gain," Electron. Lett., vol. 42, no. 9, pp. 71-72, Apr. 2006.

[3] D. A. Tulchinsky, et al., "High-saturation current widebandwidth photodetectors," IEEE J. Selected Topics Quantum Electron., vol. 10, no. 4, pp. 702-708, July/Aug. 2004.

[4] S. Thaniyavarn, Abbas, and Charczenko, "Very-low-loss electro-optic LiNbO3 components for RF/analog transmission," Avionics, Fiber-Optics and Photonics Conference, Minneapolis, paper ThC3, Sept. 2005.

[5] V. J. Urick, et al., "The performance of analog photonics links employing highly-compressed erbium-doped fiber amplifiers," IEEE Trans. Microwave Theory Tech., July 2006. 